\title{
Programme
}

\section{FRIDAY 11TH SEPTEMBER 2020}

\section{OPENING SESSION}

CET (UTC+2 Paris, Amsterdam, Berlin) 13:00 - 15:15 hours

EDT (UTC-4 New York, Montreal, Lima) 7:00 AM - 9:15 AM

CST (UTC+8 Beijing, Manila, Perth) 19:00 - 21:15 hours

\begin{tabular}{|l|l|l|}
\hline & Opening & \multicolumn{1}{|l|}{$\begin{array}{l}\text { Andrea Doria (Italy) } \\
\text { Ronald van Vollenhoven (Netherlands) }\end{array}$} \\
\hline & Keynote & \\
\hline & Moderator: Andrea Doria (Italy) & Ronald van Vollenhoven (Netherlands) \\
\hline & Understanding and treating SLE: A new era is dawning & \\
\hline \multirow{2}{*}{ Debate: New Developments in Basic Science and Clinical Research: Defining SLE } & Ricard Cervera (Spain) \\
\hline & Moderator: Ricard Cervera (Spain) & David Isenberg (UK) \\
\cline { 2 - 3 } & The matter of the debate & Murray Urowitz (Canada) \\
\cline { 2 - 3 } & A majority of lupus patients (will) need a biologic! & \\
\cline { 2 - 3 } & A minority of lupus patients (will) need a biologic! & \\
\hline
\end{tabular}

\section{PLENARY I: NEW ASPECTS IN THE MANAGEMENT OF SLE}

CET (UTC+2 Paris, Amsterdam, Berlin) 15:30 - 17:00 hours

EDT (UTC-4 New York, Montreal, Lima) 9:30 AM - 11:00 AM

CST (UTC+8 Beijing, Manila, Perth) 21:30 - 23:00 hours

\begin{tabular}{|l|l|l|}
\hline & Moderators: Zahir Amoura (France), Bernardo Pons-Estel (Argentina) & Luís Inês (Portugal) \\
\hline & Measuring SLE disease activity in 2020: Perspectives from clinical research & Chiara Tani (Italy) \\
\hline & Lifestyle for lupus patients: exercise, diet, and well-being: Perspectives from clinical practice & Ricard Cervera (Spain) \\
\hline & Microangiopathy in SLE: A diagnostic and therapeutic challenge & All \\
\hline
\end{tabular}

PLENARY II: NOVEL THERAPEUTIC DEVELOPMENTS IN 2020

CET (UTC+2 Paris, Amsterdam, Berlin) 17:30 - 19:30 hours

EDT (UTC-4 New York, Montreal, Lima) 11:30 AM - 1:30 PM

CST (UTC+8 Beijing, Manila, Perth) 23:30 - 01:30 hours

\begin{tabular}{|l|l|l|}
\hline & Moderators: Richard Furie (USA), Ronald van Vollenhoven (Netherlands) & \multicolumn{2}{l|}{ Reinhard Voll (Germany) } \\
\hline & Plasma-cell directed therapies & Bevra Hahn (USA) \\
\hline & IL-12/23 directed therapies & Thomas Dörner (Germany) \\
\hline & Novel intracellular pathways & Richard Furie (USA) \\
\hline & Molecules in early development & All \\
\hline
\end{tabular}

\section{SATURDAY 12TH SEPTEMBER 2020}

\section{PLENARY II: NOVEL THERAPEUTIC DEVELOPMENTS IN 2020}

(Recorded Session from Friday. Live Panel Discussion and Q\&A)

CET (UTC+2 Paris, Amsterdam, Berlin) 07:30 - 09:30 hours

EDT (UTC-4 New York, Montreal, Lima) 1:30 AM - 3:30 AM

CST (UTC+8 Beijing, Manila, Perth) 13:30 - 15:30 hours

\begin{tabular}{|l|l|l|}
\hline & Moderators: Richard Furie (USA), Ronald van Vollenhoven (Netherlands) & Reinhard Voll (Germany) \\
\hline & Plasma-cell directed therapies & Bevra Hahn (USA) \\
\hline & IL-12/23 directed therapies & Thomas Dörner (Germany) \\
\hline & Novel intracellular pathways & Richard Furie (USA) \\
\hline & Molecules in early development stage & All \\
\hline
\end{tabular}




\section{FOUR HOT TOPICS (SESSION 1)}

CET (UTC+2 Paris, Amsterdam, Berlin) 10:00 - 11:50 hours

EDT (UTC-4 New York, Montreal, Lima) 4.00 AM - 5.50 AM

CST (UTC+8 Beijing, Manila, Perth) 16:00 - 17:50 hours

\begin{tabular}{|l|l|l|}
\hline & Moderator: Thomas Dörner (Germany) & George Bertsias (Greece) \\
\hline & Remission and low disease activity: the new targets for treatment & Andrea Doria (Italy) \\
\hline & Belimumab helps lupus patients to achieve lupus targets & Zahir Amoura (France) \\
\hline & Can we withdraw low-dose prednisone in remitted patients? & Margherita Zen (Italy) \\
\hline & Can we withdraw immunosuppressants in remitted patients? & All \\
\hline
\end{tabular}

\section{CASE STUDY INTERACTIVE WORKSHOPS (SESSION 1)}

CET (UTC+2 Paris, Amsterdam, Berlin) 12:00 - 13:30 hours

EDT (UTC-4 New York, Montreal, Lima) 6:00 AM - 7:30 AM

CST (UTC+8 Beijing, Manila, Perth) 18:00 - 19:30 hours

\begin{tabular}{|l|l|l|}
\hline & Moderator/Facilitator: Andrea Doria (Italy) & \multicolumn{2}{|l|}{$\begin{array}{l}\text { Annegret Kuhn (Germany), } \\
\text { Marzia Caproni (Italy) }\end{array}$} \\
\hline & Management of refractory skin lupus & $\begin{array}{l}\text { Murray Urowitz (Canada), } \\
\text { Eloisa Bonfá (Brazil) }\end{array}$ \\
\hline & Moderator/Facilitator: Bernardo Pons-Estel (Argentina) & $\begin{array}{l}\text { Angela Tincani (Italy), } \\
\text { Liz Lightstone (UK) }\end{array}$ \\
\hline & Management of cardiovascular risk in SLE & \multicolumn{2}{|l|}{} \\
\hline & Fortility and pregnancy issues in patients with lupus nephritis & $\begin{array}{l}\text { Zmoura (France), } \\
\text { Richard Furie (USA) }\end{array}$ \\
\hline
\end{tabular}

\section{PRIME TIME SESSION}

CET (UTC+2 Paris, Amsterdam, Berlin) 13:30 - 15:00 hours

EDT (UTC-4 New York, Montreal, Lima) 7:30 AM - 9:00 AM

CST (UTC+8 Beijing, Manila, Perth) 19.30 - 21:00 hours

\begin{tabular}{|l|l|l|}
\hline \multicolumn{2}{|l|}{ Hot Topic } & \multicolumn{2}{l|}{} \\
\hline & Moderators: Murray Urowitz (Canada), David Isenberg (UK) & Alexandre Voskuyl (Netherlands) \\
\hline & Optimal assessment and monitoring of SLE patients in clinical practice & \\
\hline & \multicolumn{2}{|l|}{} \\
\hline & State-of-the-art & Marcello Govoni (Italy) \\
\hline & Moderators: Bevra Hahn (USA), Sandra Navarra (Philippines) & Fabrizio Conti (Italy) \\
\hline
\end{tabular}

\section{CASE STUDY INTERACTIVE WORKSHOPS (SESSION 2)}

CET (UTC+2 Paris, Amsterdam, Berlin) 15:30 - 17:00 hours

EDT (UTC-4 New York, Montreal, Lima) 9:30 AM - 11:00 AM

CST (UTC+8 Beijing, Manila, Perth) 21:30 - 23:00 hours

\begin{tabular}{|l|l|l|}
\hline & Moderator/Facilitator: Andrea Doria (Italy) & \multicolumn{2}{|l|}{$\begin{array}{l}\text { Annegret Kuhn (Germany), } \\
\text { Marzia Caproni (Italy) }\end{array}$} \\
\hline & Management of refractory skin lupus & $\begin{array}{l}\text { Murray Urowitz (Canada), } \\
\text { Eloisa Bonfá (Brazil) }\end{array}$ \\
\hline & Moderator/Facilitator: Bernardo Pons-Estel (Argentina) & Management of cardiovascular risk in SLE \\
\hline
\end{tabular}




\section{Programme}

\begin{tabular}{|l|l|l|}
\hline & Moderator/Facilitator: Sandra Navarra (Philippines) & \multicolumn{2}{|l|}{$\begin{array}{l}\text { Angela Tincani (Italy), } \\
\text { Liz Lightstone (UK) }\end{array}$} \\
\hline & Fertility and pregnancy issues in patients with lupus nephritis & $\begin{array}{l}\text { Zahir Amoura (France), } \\
\text { Richard Furie (USA) }\end{array}$ \\
\hline & Moderator/Facilitator: David Isenberg (UK) & \\
\hline
\end{tabular}

\section{FOUR HOT TOPICS (SESSION 2)}

CET (UTC+2 Paris, Amsterdam, Berlin) 17:30 - 19:40 hours

EDT (UTC-4 New York, Montreal, Lima) 11:30 AM - 1:40 PM

CST (UTC+8 Beijing, Manila, Perth) 23:30 - 01:40 hours

\begin{tabular}{|l|l|l|}
\hline & Moderator: Thomas Dörner (Germany) & George Bertsias (Greece) \\
\hline & Remission and low disease activity: the new targets for treatment & Andrea Doria (Italy) \\
\hline & Belimumab helps lupus patients to achieve lupus targets & Zahir Amoura (France) \\
\hline & Can we withdraw low-dose prednisone in remitted patients? & Margherita Zen (Italy) \\
\hline & Can we withdraw immunosuppressants in remitted patients? & All \\
\hline
\end{tabular}

\section{SUNDAY 13TH SEPTEMBER 2020}

\section{PRE-RECORDED SESSION*}

CET (UTC+2 Paris, Amsterdam, Berlin) 07:00 - 10:50 hours

EDT (UTC+4 New York, Montreal, Lima) 1:00 AM - 4.50 AM

CST (UTC+8 Beijing, Manila, Perth) 13:00 - 16:50 hours

\begin{tabular}{|l|l|l|}
\hline & Keynote & \\
\hline & Moderator: Andrea Doria (Italy) & Ronald van Vollenhoven (Netherlands) \\
\hline & $\begin{array}{l}\text { Understanding and treating SLE: A new era is dawning } \\
\text { Recorded Q\&A (15 Mins) }\end{array}$ & \\
\hline
\end{tabular}

\section{Debate: New Developments in Basic Science and Clinical Research: Defining SLE}

\begin{tabular}{|c|c|c|}
\hline & \multicolumn{2}{|l|}{ Moderator: Ricard Cervera (Spain) } \\
\hline & The matter of the debate & Ricard Cervera (Spain) \\
\hline & A majority of lupus patients (will) need a biologic! & David Isenberg (UK) \\
\hline & A minority of lupus patients (will) need a biologic! & Murray Urowitz (Canada) \\
\hline & Recorded Voting, Q\&A and Discussion (20 Mins) & All \\
\hline \multicolumn{3}{|l|}{ Hot Topic } \\
\hline & \multicolumn{2}{|l|}{ Moderators: Murray Urowitz (Canada), David Isenberg (UK) } \\
\hline & $\begin{array}{l}\text { Optimal assessment and monitoring of SLE patients in clinical practice } \\
\text { Recorded Q\&A (10 Mins) }\end{array}$ & Alexandre Voskuyl (Netherlands) \\
\hline \multicolumn{3}{|l|}{ State-of-the-art } \\
\hline & \multicolumn{2}{|l|}{ Moderators: Bevra Hahn (USA), Sandra Navarra (Philippines) } \\
\hline & $\begin{array}{l}\text { Diagnosis and treatment of neuropsychiatric lupus } \\
\text { Q\&A (10 Mins) }\end{array}$ & Marcello Govoni (Italy) \\
\hline & $\begin{array}{l}\text { Covid-19 and SLE - What do we know today? } \\
\text { Q\&A (10 Mins) }\end{array}$ & Fabrizio Conti (Italy) \\
\hline
\end{tabular}

\footnotetext{
${ }^{*}$ Please note that this session (presentation and discussion) is pre-recorded and there will not be an opportunity to participate in discussion.
} 


\section{MEET THE EDITOR}

CET (UTC+2 Paris, Amsterdam, Berlin) 11:00 - 11:55 hours

EDT (UTC+4 New York, Montreal, Lima) 5:00 AM - 5:55 AM

CST (UTC+8 Beijing, Manila, Perth) 17:00 - 17:55 hours

\begin{tabular}{|l|l|l|}
\hline & Moderator: Ronald van Vollenhoven (Netherlands) \\
\hline & Introduction: What is happening in the world of publishing? & \multirow{2}{*}{ Ronald van Vollenhoven (Netherlands) } \\
\hline & Workshop: How do I get my manuscript published_in the best possible journal? & \\
\hline & Tips for reviewers: make it work for the authors and yourself & \\
\hline
\end{tabular}

\section{POSTER SESSION}

CET (UTC+2 Paris, Amsterdam, Berlin) 12:00 - 12:50 hours

EDT (UTC+4 New York, Montreal, Lima) 6:00 AM - 6:50 AM

CST (UTC+8 Beijing, Manila, Perth) 18:00 - 18:50 hours

\begin{tabular}{|l|l|l|}
\hline Poster & Moderators: Ronald van Vollenhoven (Netherlands)/Andrea Doria (Italy) & \\
\hline & Posters: Lupus Academy Commendation & \\
& Five Posters Presentations*: (5 minutes each) & \\
& QA (5 minutes each poster) & \\
\hline
\end{tabular}

"More Posters Available Online

\section{ROUNDTABLE: REFRACTORY LUPUS MANIFESTATIONS: DEFINITION AND TREATMENT}

CET (UTC+2)Paris, Amsterdam, Berlin) 13:00 - 15:00 hours

EDT (UTC+4)New York, Montreal, Lima) 7:00 AM - 9:00 AM

CST (UTC+8 Beijing, Manila, Perth) 19:00 - 21:00 hours

\begin{tabular}{|l|l|l|}
\hline & Moderators: Andrea Doria (Italy), Ronald van Vollenhoven (Netherlands) & Sandra Navarra (Philippines) \\
\hline & $\begin{array}{l}\text { Refractory lupus nephritis } \\
\text { QA (10 Mins) }\end{array}$ & Eloisa Bonfá (Brazil) \\
\hline & $\begin{array}{l}\text { Refractory lupus cytopenias } \\
\text { QA (10 Mins) }\end{array}$ & Bernardo Pons-Estel (Argentina) \\
\hline & $\begin{array}{l}\text { Refractory musculoskeletal manifestations } \\
\text { QA (10 Mins) }\end{array}$ & Ricard Cervera (Spain) \\
\hline & $\begin{array}{l}\text { Refractory and/or catastrophic APS in SLE } \\
\text { QA (10 Mins) }\end{array}$ & All \\
\hline & Panel Discussion and Q\&A & $\begin{array}{l}\text { Andrea Doria (Italy) } \\
\text { Ronald van Vollenhoven (Netherlands }\end{array}$ \\
\hline
\end{tabular}

